\title{
THE SOLUTION OF A MINIMAX PROBLEM CONNECTED TO THE IRREDUCIBILITY OF POLYNOMIALS
}

\section{BERNT ØKSENDAL}

\section{Introduction and statement of results.}

In [2] $\mathrm{H}$. Tverberg poses the following problem: Find the value of

$$
R=\inf _{\varphi \in T_{c}}\left[\max _{x \in[\alpha, \beta]} H(x)+\max (G(-1), G(1))\right]
$$

where $T_{c}$ is the set of continuous functions $\varphi$ on $[-1,1]$ such that

$$
\varphi(-1)=\varphi(1)=0, \quad 0 \leqq \varphi \leqq 1 \quad \text { and } \quad \int_{-1}^{1} \varphi(t) d t=1 .
$$

$[\alpha, \beta]$ is the convex hull of $\operatorname{supp} \varphi$ and

$$
H(x)=\int_{-1}^{1}(1-\varphi(t)) \log |x-t| d t, \quad G(x)=\int_{-1}^{1} \varphi(t) \log |x-t| d t .
$$

The determination of $R$ is related to the following irreducibility theorem: If a polynomial $f$ of degree $n$ has integral coefficients and there are $n$ integers $a_{i}$ so that

$$
0<\left|f\left(a_{i}\right)\right|<P(n) \quad \text { for } 1 \leqq i \leqq n,
$$

then $f$ is irreducible over the rationals. The largest such number $P(n)$ is proved in [2] to have the form

$$
P(n)=\left(\lambda_{0}+o(1)\right)^{m} m ! \quad\left(m=\left[\frac{n+1}{2}\right]\right)
$$

where the constant $\lambda_{0}$ equals $\exp \left(1+\frac{1}{2} R\right)$.

We shall prove that there exists a unique function $\varphi_{0}$ which is optimal for the problem (1.1). The function $\varphi_{0}$ is given by 


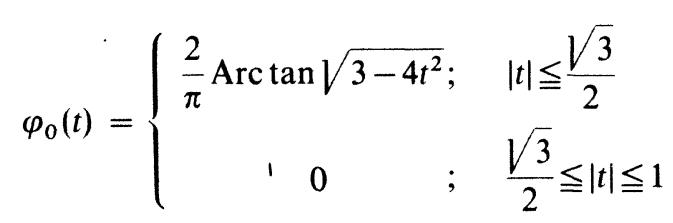

The corresponding minimal value is $R=-2+4 \log 2-\frac{3}{2} \log 3$. (This solution was anticipated in [2]). Hence the value of $\lambda_{0}$ is $\frac{4}{3} \cdot 3^{\frac{1}{4}}=1.754 \ldots$.

We will proceed as follows. After reformulating the problem slightly, we first prove that there exists at least one optimal function in $L^{\infty}[-1,1]$ for the problem (Lemma 1). We then prove that for such an optimal function $\varphi$ the function

$$
H_{\varphi}(x)=H(x)=\int_{-1}^{1}(1-\varphi(t)) \log |x-t| d t
$$

must be constant on the convex hull $[\alpha, \beta]$ of $\operatorname{supp} \varphi$ (Theorem 1). This reduces the problem to solving an integral equation $H_{\varphi}(x)=$ constant on $[\alpha, \beta]$. Using known inversion formulas for the Hilbert transform it is finally proved that the only bounded solution $\varphi$ of this integral equation is the function $\varphi_{0}$ above (Theorem 2).

The author is greatly indebted to $H$. Tverberg for many valuable conversations. Theorem 2 is due to him.

\section{Existence of optimal functions.}

Put

$$
T=\left\{\varphi \in L^{\infty}[-1,1] ; 0 \leqq \varphi \leqq 1, \int_{-1}^{1} \varphi(t) d t=1\right\}
$$

Define

$$
G_{\varphi}(x)=\int_{-1}^{1} \varphi(t) \log |x-t| d t
$$

and

$$
R_{\varphi}=\max _{x \in[\alpha, \beta]} G_{1-\varphi}(x)+\max \left(G_{\varphi}(-1), G_{\varphi}(1)\right), \quad \text { for } \varphi \in T,
$$

where $[\alpha, \beta]$ is the convex hull of $\operatorname{supp} \varphi$.

We will replace the family $T_{c}$ by the larger family $T$ and consider

$$
R_{1}=\inf \left\{R_{\varphi} ; \varphi \in T\right\} \text {. }
$$


It turns out that the optimal function for this problem is the function $\varphi_{0}$ above, which is a member of $T_{c}$. Therefore $R=R_{1}$, so that

$$
R=\inf \left\{R_{\varphi} ; \varphi \in T\right\} \text {. }
$$

However, (2.1) can also be deduced directly as follows:

If $\varphi \in T$ we can find a sequence $\left\{\varphi_{n}\right\} \subset T_{c}$ converging to $\varphi$ weakstar in $L^{\infty}[-1,1]$ such that $\operatorname{supp} \varphi_{n} \subseteq[\alpha, \beta]$, the convex hull of $\operatorname{supp} \varphi$. Then $G_{\varphi_{n}}(x) \rightarrow G_{\varphi}(x)$ for all $x \in[-1,1]$. Since $\left\{G_{\varphi_{n}}\right\}$ is equicontinuous on $[-1,1]$ (because $0 \leqq \varphi_{n} \leqq 1$ ), we get by the Ascoli theorem that $G_{\varphi_{n}} \rightarrow G_{\varphi}$ uniformly on $[-1,1]$. Therefore

$$
\begin{aligned}
R_{\varphi} & =\max _{x \in[\alpha, \beta]} G_{1-\varphi}(x)+\max \left(G_{\varphi}(-1), G_{\varphi}(1)\right) \\
& =\lim _{n \rightarrow \infty}\left[\max _{x \in[\alpha, \beta]} G_{1-\varphi_{n}}(x)+\max \left(G_{\varphi_{n}}(-1), G_{\varphi_{n}}(1)\right)\right] \geqq \lim _{n \rightarrow \infty} R_{\varphi_{n}},
\end{aligned}
$$

which proves (2.1).

Next, observe that if we put $\psi=1-\varphi$ we have $\psi \in T$ if $\varphi \in T$ and

$$
\begin{aligned}
G_{\psi}(x) & =\int_{-1}^{1} \log |x-t| d t-G_{\varphi}(x) \\
& =(1-x) \log (1+x)+(1+x) \log (1+x)-2-G_{\varphi}(x) .
\end{aligned}
$$

Hence

$$
\begin{aligned}
G_{\psi}(-1) & =2 \log 2-2-G_{\varphi}(-1) \\
G_{\psi}(1) & =2 \log 2-2-G_{\varphi}(1) .
\end{aligned}
$$

Therefore, if we put

$$
\begin{gathered}
N_{\psi}=\max _{[\alpha, \beta]} G_{\psi}(x)-\min \left(G_{\psi}(-1), G_{\psi}(1)\right) ;[\alpha, \beta]=\operatorname{conv}(\operatorname{supp}(1-\psi)) \\
N=\inf \left\{N_{\psi} ; \psi \in T\right\}
\end{gathered}
$$

we have

$$
N_{\psi}=R_{\varphi}+2-2 \log 2 \quad \text { and } \quad N=R+2-2 \log 2 .
$$

Therefore we proceed to work with the problem (2.3). A result similar to the following lemma is mentioned in Tverberg [2, p. 14]. Since it is so crucial for our approach to the problem we state it again and include a proof:

Lemma 1. There exists a function $\psi_{0} \in T$ which is optimal for the problem (2.3). that is, $N_{\psi_{0}}=N$. 
Proof. Let $\left\{\psi_{n}\right\}_{n=1}^{\infty} \subset T$ be a sequence such that $N_{\psi_{n}} \rightarrow N$. Since the unit ball in $L^{\infty}[-1,1]$ is compact in the weak-star topology, there exists a subnet $\left\{\psi_{i}\right\}_{i \in I}$ converging weak-star to a function $\psi_{0} \in L^{\infty}[-1,1]$. Since the weak-star topology on the unit ball of $L^{\infty}[-1,1]$ is metrizable, we can replace the subnet by a subsequence which we again denote by $\left\{\psi_{n}\right\}_{n=1}^{\infty}$.

We have

$$
\int_{-1}^{1} f(t) \psi_{n}(t) d t \rightarrow \int_{-1}^{1} f(t) \psi_{0}(t) d t \quad \text { as } n \rightarrow \infty
$$

for all $f \in L^{1}[-1,1]$. This implies that

$$
0 \leqq \psi_{0} \leqq 1, \quad \int_{-1}^{1} \psi_{0}(t) d t=1,
$$

so that $\psi_{0} \in T$. Moreover, turning to a subsequence if necessary we can assume that $\alpha_{n} \rightarrow \alpha_{0}, \beta_{n} \rightarrow \beta_{0}$, where $\left[\alpha_{n}, \beta_{n}\right]$ is the convex hull of supp $\left(1-\psi_{n}\right)$. Then $\operatorname{supp}\left(1-\psi_{0}\right) \subset\left[\alpha_{0}, \beta_{0}\right]$. Since $G_{\psi_{n}}(x) \rightarrow G_{\psi_{0}}(x)$ for all $x \in[-1,1]$, we get using the Ascoli theorem again that $G_{\psi_{n}} \rightarrow G_{\psi_{0}}$ uniformly on $[-1,1]$. Hence

$$
\begin{aligned}
N \leqq N_{\psi_{0}} & =\max _{\operatorname{conv}\left(\operatorname{supp}\left(1-\psi_{0}\right)\right)} G_{\psi_{0}}(x)-\min \left(G_{\psi_{0}}(-1), G_{\psi_{0}}(1)\right) \\
& \leqq \max _{\left[\alpha_{0}, \beta_{0}\right]} G_{\psi_{0}}(x)-\min \left(G_{\psi_{0}}(-1), G_{\psi_{0}}(1)\right) \\
& =\lim _{n \rightarrow \infty} N_{\psi_{n}}=N .
\end{aligned}
$$

We conclude that $N_{\psi_{0}}=N$ and the proof is complete.

\section{Proof that if $\psi$ is optimal then $G_{\psi}$ must be constant on $[\alpha, \beta]$.}

We will prove this using a variational technique. The idea is simple: If $G_{\psi}$ is not constant on $[\alpha, \beta]$, we modify $\psi$ slightly - by adding and subtracting suitably - to obtain a function $\psi_{1} \in T$ such that $N_{\psi_{1}}<N_{\psi}$. The idea is to add a little to $\psi$ at points where $G_{\psi}$ is big (thereby reducing $G_{\psi}$ near these points) and subtract from $\psi$ accordingly at a point where $G_{\psi}$ is small.

The next three lemmas enables us to carry out these modifications on $\psi$ such that the modified function $\psi_{1}$ still belongs to $T$.

Lemma 2. Let $\psi \in T$. If $\psi=1$ a.e. in a neighbourhood of $c \in(-1,1)$, then $G_{\psi}$ is twice continuously differentiable at $c$ and $G_{\psi}^{\prime \prime}(c)>0$.

Proof. Tverberg [2, page 19].

Lemma 3. Let $\psi \in T$. If $\psi=0$ a.e. in a neighbourhood of $c \in(-1,1)$, then $G_{\psi}$ is twice continuously differentiable at $c$ and $G_{\psi}^{\prime \prime}(c)<0$. 
Proof. As in Tverberg [2, page 19], we get

$$
G_{\psi}^{\prime \prime}(c)=-\left(\int_{-1}^{c-\varepsilon}+\int_{c+\varepsilon}^{1}\right) \psi(t) \frac{d t}{(c-t)^{2}}<0 .
$$

Immediate consequences of lemmas 2 and 3 are:

Lemma 4. (i) Let $x_{0}$ be a local minimum point for $G_{\psi}$ in $[\alpha, \beta] \subset(-1,1)$. Then

$$
\int_{J} \psi(t) d t>0 \text { for every open interval } J \ni x_{0} \text {. }
$$

(ii) Let $x_{1}$ be a local maximum point for $G_{\psi}$ in $[\alpha, \beta] \subset(-1,1)$. Then

$$
\int_{J}(1-\psi(t)) d t>0 \quad \text { for every open interval } J \ni x_{1} .
$$

Hence if $G_{\psi}$ is not constant on $[\alpha, \beta]$ it is possible to add something to $\psi$ near maximum points and subtract near minimum points such that the modified function still belongs to $T$. However, it is not clear that this can be done such that $N_{\psi}$ is reduced. For this we need some technical lemmas:

LEMMA 5 . Let $0 \leqq a<b \leqq 1$. Then

$$
\frac{\log (1-a)}{\log (1-b)}<\frac{a}{b}<\frac{\log (1+a)}{\log (1+b)} \text {. }
$$

Proof. Using Cauchy's mean value theorem for a quotient we get

$$
\frac{\log (1+x a)-\log (1+0 \cdot a)}{\log (1+x b)-\log (1+0 \cdot b)}=\frac{\frac{a}{1+y a}}{\frac{b}{1+y b}}=\frac{a}{b} \cdot \frac{1+y b}{1+y a}
$$

with $y$ strictly between 0 and $x$. Putting $x= \pm 1$ we get the lemma.

Lemma 6. Assume $-1<x_{1}<x_{0}<x_{2}<1$. Define

$$
f_{\lambda}(x)=\lambda \log \left|x-x_{1}\right|+(1-\lambda) \log \left|x-x_{2}\right|-\log \left|x-x_{0}\right|
$$

for $x \in[-1,1], \lambda \in[0,1]$. Choose $\lambda_{0} \in(0,1)$ such that $x_{0}=\lambda_{0} x_{1}+\left(1-\lambda_{0}\right) x_{2}$.

Then we have:

(I) $f_{\lambda}^{\prime}(x)<0$ for all $x \in\left[-1, x_{1}\right) \Leftrightarrow \lambda_{0} \frac{1+x_{1}}{1+x_{0}}<\lambda$. 
(II) $f_{\lambda}^{\prime}(x)>0$ for all $x \in\left(x_{2}, 1\right] \Leftrightarrow \lambda<\lambda_{0} \frac{1-x_{1}}{1-x_{0}}$.

(III)

$$
f_{\lambda}(-1)=f_{\lambda}(1) \Leftrightarrow \lambda=\bar{\lambda}=\log \frac{\left(1-x_{2}\right)\left(1+x_{0}\right)}{\left(1+x_{2}\right)\left(1-x_{0}\right)} / \log \frac{\left(1-x_{2}\right)\left(1+x_{1}\right)}{\left(1+x_{2}\right)\left(1-x_{1}\right)} .
$$

(IV) $\lambda_{0} \frac{1+x_{1}}{1+x_{0}}<\pi<\lambda_{0} \frac{1-x_{1}}{1-x_{0}}$.

Proof. (I): Let $-1 \leqq x<x_{1}$. Then

$$
f_{\lambda}^{\prime}(x)=\frac{\lambda}{x-x_{1}}+\frac{1-\lambda}{x-x_{2}}-\frac{1}{x-x_{0}}=\frac{\left(x_{1}-x\right)\left(x_{2}-x\right)-\left(x_{0}-x\right)\left(x_{3}-x\right)}{\left(x_{0}-x\right)\left(x_{1}-x\right)\left(x_{2}-x\right)},
$$

where $x_{3}=\lambda x_{2}+(1-\lambda) x_{1}$. Put

$$
g(x)=\left(x_{1}-x\right)\left(x_{2}-x\right)-\left(x_{0}-x\right)\left(x_{3}-x\right) .
$$

Then we see that

$$
\begin{aligned}
f_{\lambda}^{\prime}(x)<0 & \Leftrightarrow g(x)<0 \\
& \Leftrightarrow x_{3}>x+\frac{\left(x_{1}-x\right)\left(x_{2}-x\right)}{x_{0}-x} \\
& \Leftrightarrow \lambda>\frac{\left(x_{2}-x_{0}\right)\left(x_{1}-x\right)}{\left(x_{2}-x_{1}\right)\left(x_{0}-x\right)}=\lambda_{0} \frac{x_{1}-x}{x_{0}-x} .
\end{aligned}
$$

The last inequality holds for all $x \in\left[-1, x_{1}\right]$ if and only if $\lambda>\lambda_{0}\left(1+x_{1}\right) /$ $\left(1+x_{0}\right)$.

(II): If we apply (I) to $f_{1-\lambda}$ with $x_{1}, x_{0}, x_{2}, \lambda_{0}$ replaced by $-x_{2},-x_{0},-x_{1}$, $1-\lambda_{0}$, we obtain (II).

(III): This is straightforward.

(IV): If we substitute $x_{0}=\lambda_{0} x_{1}+\left(1-\lambda_{0}\right) x_{2}$ we see that

$$
\frac{\left(1-x_{2}\right)\left(1+x_{0}\right)}{\left(1+x_{2}\right)\left(1-x_{0}\right)}=1-\frac{2 \lambda_{0}\left(x_{2}-x_{1}\right)}{\left(1+x_{2}\right)\left(1-x_{0}\right)} \text {. }
$$

Similarly

$$
\frac{\left(1-x_{2}\right)\left(1+x_{1}\right)}{\left(1+x_{2}\right)\left(1-x_{1}\right)}=1-\frac{2\left(x_{2}-x_{1}\right)}{\left(1+x_{2}\right)\left(1-x_{1}\right)} .
$$

Hence by lemma 5

$$
\pi<\lambda_{0} \frac{1-x_{1}}{1-x_{0}}
$$


To obtain the other inequality, we rewrite $\bar{\lambda}$ as

$$
\bar{\lambda}=\frac{\log \frac{\left(1+x_{2}\right)\left(1-x_{0}\right)}{\left(1-x_{2}\right)\left(1+x_{0}\right)}}{\log \frac{\left(1+x_{2}\right)\left(1-x_{1}\right)}{\left(1-x_{2}\right)\left(1+x_{1}\right)}}=\frac{\log \left(1+\frac{2 \lambda_{0}\left(x_{2}-x_{1}\right)}{\left(1-x_{2}\right)\left(1+x_{0}\right)}\right)}{\log \left(1+\frac{2\left(x_{2}-x_{1}\right)}{\left(1-x_{2}\right)\left(1+x_{1}\right)}\right)}>\lambda_{0} \frac{1+x_{1}}{1+x_{0}}
$$

again by lemma 5 .

We are now ready for the main result in this section:

THEOREM 1. Let $\psi$ be a function in $T$ such that $N_{\psi}=N$. Then

$$
G_{\psi}(x) \text { is constant on }[\alpha, \beta] \text {, }
$$

where as before $[\alpha, \beta]$ is the convex hull of $\operatorname{supp}(1-\psi)$.

Proof. In [2] it is proved that the function $\varphi_{0}$ mentioned in the introduction gives

$$
N_{1-\varphi_{0}}=2 \log 2-\frac{2}{3} \log 3<0 .
$$

Hence if $\psi$ is optimal we have $N_{\psi}<0$ and therefore $[\alpha, \beta] \subset(-1,1)$.

Assume $G_{\psi}(x)$ is not constant on $[\alpha, \beta]$. Then

$$
m=\min _{[\alpha, \beta]} G_{\psi}(x)<\max _{[\alpha, \beta]} G_{\psi}(x)=M .
$$

The easier case, when $G_{\psi}(\alpha)=m$, or $G_{\psi}(\beta)=m$, will be dealt with afterwards.

For the moment we choose an $x_{0} \in(\alpha, \beta)$ so that $G_{\psi}\left(x_{0}\right)=m$. Put

$$
\begin{aligned}
& M_{1}=\max \left\{G_{\psi}(x) ; x \in\left[\alpha, x_{0}\right]\right\} \\
& M_{2}=\max \left\{G_{\psi}(x) ; x \in\left[x_{0}, \beta\right]\right\} .
\end{aligned}
$$

Then $M_{1}>m, M_{2}>m$. Let furthermore

$$
\begin{aligned}
& x_{1}=\max \left\{x \in\left[\alpha, x_{0}\right] ; G_{\psi}(x)=M_{1}\right\} \\
& x_{2}=\min \left\{x \in\left[x_{0}, \beta\right] ; G_{\psi}(x)=M_{2}\right\} .
\end{aligned}
$$

Then $-1<\alpha \leqq x_{1}<x_{0}<x_{2} \leqq \beta<1$.

Let $\delta>0$ and let $J_{0}, J_{1}, J_{2}$ be disjoint relatively open intervals in $[\alpha, \beta]$ of length $2 \delta$ centered at $x_{0}, x_{1}, x_{2}$, respectively. Then by lemma 4 we can find positive numbers $u, v, w \leqq 1$ such that

$$
u \int_{J_{1}}(1-\psi(t)) d t=v \int_{J_{2}}(1-\psi(t)) d t=w \int_{J_{0}} \psi(t) d t=\varepsilon>0 .
$$


Now put

$$
\Delta(t)=\left\{\begin{array}{lll}
\bar{\lambda} u(1-\psi(t)) & ; & t \in J_{1} \\
-w \psi(t) & ; & t \in J_{0} \\
(1-\bar{\lambda}) v(1-\psi(t)) ; & t \in J_{2} \\
0 & \text { otherwise }
\end{array}\right.
$$

where $\bar{\lambda}$ is the quantity of lemma 6 . Then clearly, as $0<\bar{\lambda}<1$,

$$
0 \leqq \psi(t)+\Delta(t) \leqq 1 \quad \text { for all } t \in[-1,1] .
$$

Since

$$
\int_{-1}^{1} \Delta(t) d t=\bar{\lambda} \varepsilon-\varepsilon+(1-\bar{\lambda}) \varepsilon=0
$$

we have $\psi+\Delta \in T$. Moreover $\Delta=0$ outside $[\alpha, \beta]$.

Now consider

$$
\begin{aligned}
G_{\Delta}(x)= & \left(\int_{J_{1}}+\int_{J_{2}}+\int_{J_{0}}\right) \Delta(t) \log |x-t| d t \\
= & \bar{\lambda} \int_{J_{1}} u(1-\psi(t)) \log |x-t| d t+(1-\bar{\lambda}) \int_{J_{2}} v(1-\psi(t)) \log |x-t| d t \\
& -\int_{J_{0}} w \psi(t) \log |x-t| d t \\
= & \varepsilon\left(\bar{\lambda} \log \left|x-t_{1}\right|+(1-\bar{\lambda}) \log \left|x-t_{2}\right|-\log \left|x-t_{0}\right|\right),
\end{aligned}
$$

where $t_{i} \in J_{i}$ depend on $x$.

Let $U_{0}, U_{1}, U_{2}$ be disjoint open intervals centered at $x_{0}, x_{1}, x_{2}$ respectively. Then as $\delta \rightarrow 0$,

$$
\frac{1}{\varepsilon} G_{\Delta}(x) \rightarrow f_{\lambda}(x) \quad \text { uniformly on }[-1,1] \backslash \bigcup_{i=0}^{2} U_{i},
$$

where $f_{\bar{\lambda}}$ is the function from lemma 6 . Therefore, if we put

$$
\Lambda=\min \left(G_{\Delta}(-1), G_{\Delta}(1)\right), \quad U_{i}=\left(r_{i}, s_{i}\right) ; \quad 0 \leqq i \leqq 2,
$$

we have for sufficiently small $\delta$ that

(i) $\max \left\{G_{\Delta}(x) ; x \in\left[\alpha, r_{1}\right] \cup\left[s_{2}, \beta\right]\right\}<\Lambda$.

Choose positive numbers $\sigma_{1}, \sigma_{2}$ such that $x_{1}+\sigma_{1}<x_{2}-\sigma_{2}$ and

$$
\begin{aligned}
& x_{1}<x<x_{1}+\sigma_{1} \Rightarrow f_{\lambda}(x)<f_{\lambda}(-1) \\
& x_{2}-\sigma_{2}<x<x_{2} \Rightarrow f_{\lambda}(x)<f_{\lambda}(1) .
\end{aligned}
$$


Then if $\delta$ is small enough, we obtain

(ii) $\max \left\{G_{\Delta}(x) ; x \in\left[x_{1}, x_{1}+\sigma_{1}\right] \cup\left[x_{2}-\sigma_{2}, x_{2}\right] \backslash \bigcup_{i=0}^{2} U_{1}\right\}<\Lambda$.

(Note that $\sigma_{1}, \sigma_{2}$ are independent of $\delta$ ).

Finally if $x \in U_{1}$ we have

(iii) $G_{\Delta}(x)<\varepsilon\left(\bar{\lambda} \log \left(s_{1}-r_{1}\right)+\log 2-\log \left(r_{0}-s_{1}\right)\right)<\Lambda$,

if $s_{r}-r_{1}$ is chosen small enough .

Similarly for $x \in U_{2}$.

Summing up, we conclude from (i), (ii) and (iii) that

(a) $G_{\Delta}(x)<\Lambda$ for all $x \in\left[\alpha, x_{1}+\sigma_{1}\right] \cup\left[x_{2}-\sigma_{2}, \beta\right]$, for sufficiently small $\delta$.

Furthermore, choosing $\delta$ small enough, we can obtain

(b) $\bar{m}+\max \left\{\left|G_{\Delta}(x)\right| ; x \in\left[x_{1}+\sigma_{1}, x_{2}-\sigma_{2}\right]\right\}$

$<M-\max \left\{\left|G_{\Delta}(x)\right| ; x \in[-1,1]\right\}$,

where $\bar{m}=\max \left\{G_{\psi}(x) ; x \in\left[x_{1}+\sigma_{1}, x_{2}-\sigma_{2}\right]\right\}$.

Now consider $G_{\psi+\Delta}(x)=G_{\psi}(x)+G_{\Delta}(x)$. From (a) and (b) we have

$$
\begin{aligned}
\max _{[\alpha, \beta]} G_{\psi+\Delta}(x) & =\max _{\left[\alpha, x_{1}+\sigma_{1}\right] \cup\left[x_{2}-\sigma_{2}, \beta\right]}\left(G_{\psi}(x)+G_{\Delta}(x)\right\} \\
& <\max _{[\alpha, \beta]} G_{\psi}(x)+\Lambda .
\end{aligned}
$$

Therefore,

$$
\begin{aligned}
N_{\psi+\Delta} & =\max _{[\alpha, \beta]}\left(G_{\psi+\Delta}(x)\right)-\min \left(G_{\psi+\Delta}(-1), G_{\psi+\Delta}(1)\right) \\
& <\max _{[\alpha, \beta]} G_{\psi}(x)+\Lambda-\min \left(G_{\psi}(-1), G_{\psi}(1)\right)-\min \left(G_{\Delta}(-1), G_{\Delta}(1)\right)=N_{\psi} .
\end{aligned}
$$

This contradiction proves the theorem for the case that $G_{\psi}(\alpha) \neq m, G_{\psi}(\beta) \neq m$.

In the case when, say, $G_{\psi}(\beta)=m$, we put $x_{0}=\beta$ and proceed as above except we use the value $\bar{\lambda}=1$. So in this case we define

$$
\Delta(t)=\left\{\begin{array}{cl}
u(1-\psi(t)) ; & t \in J_{1} \\
-w \psi(t) ; & t \in J_{0} \\
0 & \text { otherwise }
\end{array}\right.
$$

The proof of the necessary inequalities proceed similarly, except they are easier to establish in this case. This completes the proof of theorem 1. 


\section{The solution of the integral equation.}

The last step in the solution of our minimax problem consists of solving the integral equation that an optimal $\psi$ must satisfy, according to theorem 1 .

THEOREM 2 (Tverberg). Let $\varphi \in T$ satisfy

$$
\int_{-1}^{1}(1-\varphi(t)) \log |x-t| d t=K \quad \text { for } x \in[\alpha, \beta]
$$

where $[\alpha, \beta]$ is the convex hull of $\operatorname{supp} \varphi$ and $K$ is a constant.

Then $[\alpha, \beta]=\left[-\frac{1}{2} \sqrt{3}, \frac{1}{2} \sqrt{3}\right\}$ and

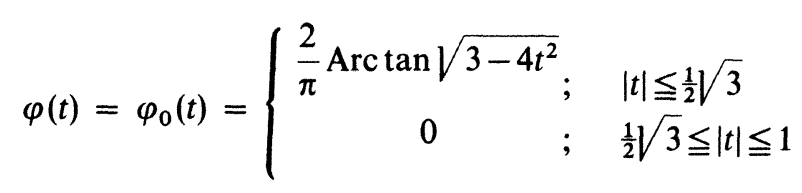

Proof. Differentiating (4.1) we get

$$
\int_{\alpha}^{\beta} \frac{\varphi(t)}{x-t} d t=\log (1+x)-\log (1-x), \quad x \in[\alpha, \beta],
$$

where we take the Cauchy principal value of the integral, that is,

$$
\lim _{\varepsilon \rightarrow 0}\left(\int_{\alpha}^{x-\varepsilon}+\int_{x+\varepsilon}^{\beta}\right)
$$

The general solution of the equation (4.2) is given by

$$
\pi^{2} \sqrt{(x-\alpha)(\beta-x)} \varphi(x)=\int_{\alpha}^{\beta} \frac{\sqrt{(t-\alpha)(\beta-t)}}{t-x} \log \frac{1+t}{1-t} d t+\pi C,
$$

where $C=\int_{\alpha}^{\beta} \varphi(t) d t$ (see $[1$, p. 178]). Thus in our case $C=1$. As $\varphi \leqq 1$, the limit of the right hand side of (4.3) is 0 as $x$ goes to $\alpha$ or $\beta$. Thus, as is easy to see,

$$
\int_{\alpha}^{\beta} \sqrt{\frac{\beta-t}{t-\alpha}} \log \frac{1+t}{1-t} d t=-\int_{\alpha}^{\beta} \sqrt{\frac{t-\alpha}{\beta-t}} \log \frac{1+t}{1-t} d t=-\pi
$$

Replacing $t$ by $\alpha+\beta-t$ in the second integral of (4.4), we conclude

$$
\int_{\alpha}^{\beta} \sqrt{\frac{\beta-t}{t-\alpha}} \log \frac{(1+t)(1+\alpha+\beta-t)}{(1-t)(1-\alpha-\beta+t)} d t=0 .
$$

Thus $\alpha+\beta=0$, since the integrand has the same sign as $\alpha+\beta$. Therefore we have from (4.4)

$$
\beta \int_{0}^{1}\left(\sqrt{\frac{1+t}{1-t}}-\sqrt{\frac{1-t}{1+t}}\right) \log \frac{1+\beta t}{1-\beta t} d t=\pi
$$


Since the integrand increases with $\beta$, we conclude that $\beta$, and hence $\varphi$, is unique. The function $\varphi_{0}$ defined in the introduction satisfies

$$
0 \leqq \varphi_{0} \leqq 1, \quad \int_{-1}^{1} \varphi(\mathrm{t}) \mathrm{dt}=1
$$

And it was shown in [2, pp. 15-16], that $\varphi_{0}$ satisfies the equation (4.1), with $K$ $=-1+\frac{3}{2} \log 3-\log 4$. So we must have $\varphi=\varphi_{0}$ and the proof is complete.

Thus the function $\varphi_{0}$ is the solution to our problem. It follows that the minimum value $N=N_{1-\varphi_{0}}$ is $2 \log 2-\frac{3}{2} \log 3$. This gives

$$
R=-2+4 \log 2-\frac{3}{2} \log 3,
$$

and

$$
\lambda_{0}=\exp \left(1+\frac{1}{2} R\right)=\frac{4}{3} \cdot 3^{\frac{1}{4}}
$$

as mentioned in the introduction.

\title{
REFERENCES
}

1. F. G. Tricomi, Integral equations, Interscience Publishers, London, New York, 1957.

2. H. Tverberg, On the irreducibikity of polynomials taking small values, Math. Scand. 32 (1971), 5-21.

\author{
AGDER DISTRIKTSHøGSKOLE \\ POSTBOX 607 \\ 4601 KRISTIANSAND S \\ NORWAY
}

